\title{
Modeling Short-Pulse-Driven Collisional $X$-Ray Lasers and Other New Schemes
}

\author{
J. Nilsen \\ Y. Li \\ J. Dunn \\ A.L. Osterheld \\ This paper was prepared for submittal to the \\ 1999 International Symposium on Optical Science, Engineering, and Instrumentation \\ Denver, $\mathrm{CO}$ \\ July 18-23, 1999
}

July 1, 1999

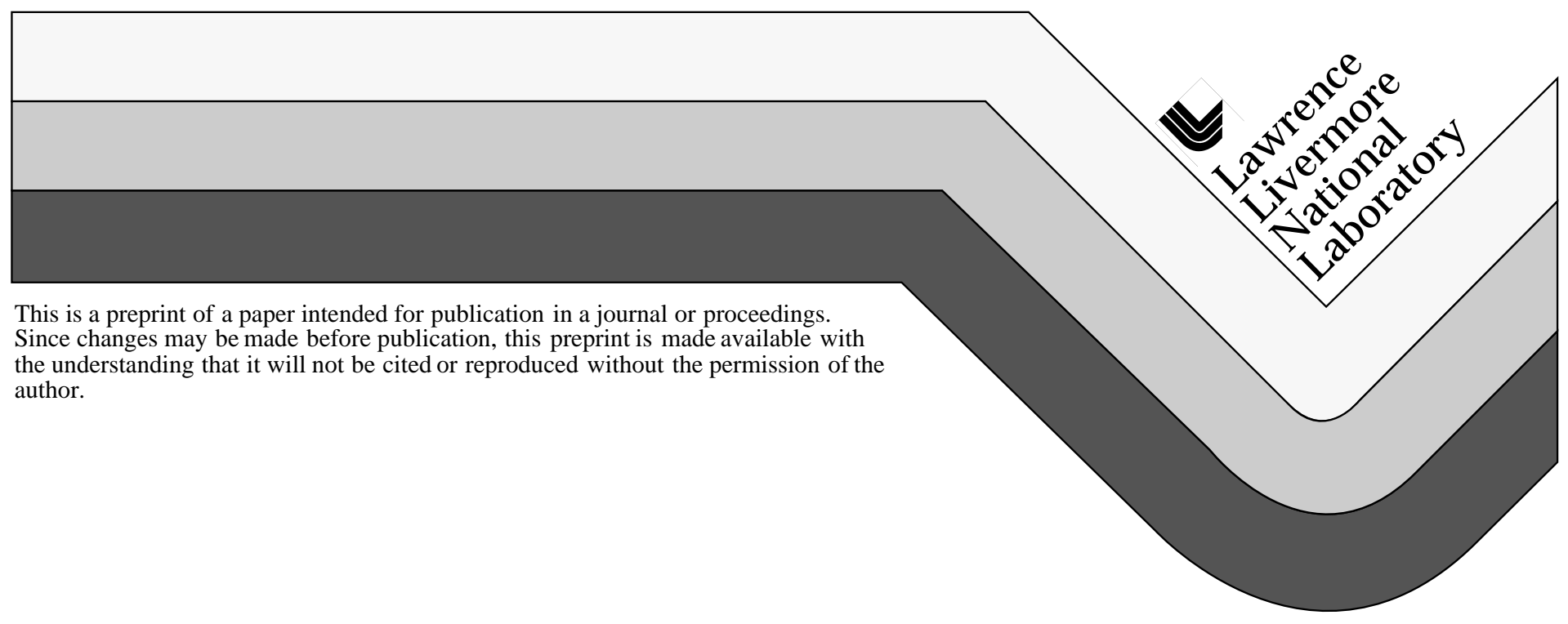




\section{DISCLAIMER}

This document was prepared as an account of work sponsored by an agency of the United States Government. Neither the United States Government nor the University of California nor any of their employees, makes any warranty, express or implied, or assumes any legal liability or responsibility for the accuracy, completeness, or usefulness of any information, apparatus, product, or process

disclosed, or represents that its use would not infringe privately owned rights. Reference herein to any specific commercial product, process, or service by trade name, trademark, manufacturer, or otherwise, does not necessarily constitute or imply its endorsement, recommendation, or favoring by the United States Government or the University of California. The views and opinions of authors expressed herein do not necessarily state or reflect those of the United States Government or the University of California, and shall not be used for advertising or product endorsement purposes. 


\title{
Modeling short-pulse-driven collisional X-ray lasers and other new schemes
}

\author{
Joseph Nilsen ${ }^{\mathrm{a}}$, Yuelin Li ${ }^{\mathrm{b}}$, James Dunn ${ }^{\mathrm{a}}$, and Albert L. Osterheld ${ }^{\mathrm{a}}$ \\ ${ }^{a}$ Lawrence Livermore National Laboratory, Livermore, CA 94550 \\ ${ }^{\mathrm{b}}$ Institute for Laser Science and Applications, Lawrence Livermore National Laboratory, Livermore, CA
}

\begin{abstract}
Recently, the technique of using a nsec pulse to preform and ionize the plasma followed by a psec pulse to heat the plasma has enabled low-Z neon-like and nickel-like ions to lase driven by small lasers with only ten joules of energy. In this work we model recent experiments done using the COMET laser at LLNL to illuminate $1 \mathrm{~cm}$ long slab targets of Ti with a $4.8 \mathrm{~J}, 800$ ps prepulse followed $1.6 \mathrm{nsec}$ later by a $6 \mathrm{~J}, 1 \mathrm{psec}$ drive pulse. The LASNEX code is used to calculate the hydrodynamic evolution of the plasma and provide the temperatures and densities to the XRASER code, which then does the kinetics calculations to determine the gain. The temporal and spatial evolution of the plasma is studied both with and without radiation transport included for the $3 \mathrm{~d}$ and $3 \mathrm{~s} \_2 \mathrm{p}$ Ne-like Ti resonance lines. Large regions with gains greater than $80 \mathrm{~cm}^{-1}$ are predicted for the $3 \mathrm{p}^{1} \mathrm{~S}_{0} \ldots 3 \mathrm{~s}{ }^{1} \mathrm{P}_{1} \mathrm{Ne}$-like Ti laser line at $326 \AA$. Given the large gain and large gradients in these plasmas, we do propagation calculations including refraction to understand which regions have the right combination of high gain and low gradients to contribute to the X-ray laser output. Calculations are also presented using different delays between the long and short pulse and different widths for the short pulse to provide better insight for optimizing the laser output. In addition to the standard $326 \AA$ laser line, high gain is also predicted and observed for the $3 \mathrm{~d}{ }^{1} \mathrm{P}_{1}-3 \mathrm{p}^{1} \mathrm{P}_{1}$ laser line at $301 \AA$ in Ne-like Ti. We present calculations with and without radiation transport included on the strong $3 \mathrm{~d}^{1} \mathrm{P}_{1}-{ }^{2} \mathrm{p}{ }^{1} \mathrm{~S}_{0}$ resonance line to better understand this self photopumping effect. We also look at the analog transition in Ni-like ions to understand if self photopumping may also play a role in Ni-like ions. High gain is predicted on the $3 \mathrm{~d}^{9} 4 \mathrm{f}^{1} \mathrm{P}_{1}-3 \mathrm{~d}^{9} 4 \mathrm{~d}{ }^{1} \mathrm{P}_{1}$ Ni-like transition and this line has recently been observed at $226 \AA$ in Ni-like Mo. Gain of $13 \mathrm{~cm}^{-1}$ has been measured for this line.
\end{abstract}

Keywords: X-ray laser, multiple pulse technique

\section{INTRODUCTION}

Most researchers today use some variant of the prepulse or multiple pulse technique [1-3] to achieve lasing in Ne-like or $\mathrm{Ni}$-like ions. As a result the Ne-like $3 \mathrm{p}^{1} \mathrm{~S}_{0} \ldots 3 \mathrm{~s}{ }^{1} \mathrm{P}_{1}$ laser line now dominates the laser output as was predicted two decades ago. These techniques illuminate solid targets with several pulses. The first pulse is used to create a large scale length plasma which is in the correct density range for gain and has sufficiently small density gradients for laser propagation. The subsequent pulses are then absorbed more efficiently and heat the plasma to lasing conditions. Typical pulse durations are $100 \mathrm{ps}$ for the multiple pulse technique and up to $1 \mathrm{~ns}$ for the prepulse technique and typically use 100 to $1000 \mathrm{~J}$ of energy. In the above techniques the pulse duration is held constant but the contrast and separation between pulses is varied. A recent variant of these techniques, which has now become very common, is the use of a nsec prepulse followed by a psec drive pulse. This approach was first used to demonstrate lasing at $326 \AA$ in Ne-like Ti at the Max Born Institute (MBI) [4] using less than 10 joules of energy. The Ti experiments were reproduced at Lawrence Livermore National Laboratory (LLNL) using the COMET laser and extended to Ni-like Pd at $146 \AA$ [5] and very recently to Ni-like Mo at $189 \AA$ [6]. In this paper we model the Ne-like Ti experiments done with the COMET laser to understand the plasma conditions present and what gain is possible on different transitions. In addition to the Ne-like Ti $3 \mathrm{p}^{1} \mathrm{~S}_{0}-3 \mathrm{~s}^{1} \mathrm{P}_{1}$ transition at $326 \AA$ we discuss the photopumping mechanism [7,8] which drives the gain of the Ne-like Ti $3 \mathrm{~d}^{1} \mathrm{P}_{1} \ldots 3 \mathrm{p}^{1} \mathrm{P}_{1}$ transition at $301 \AA$. For Ni-like Mo we present lasing on both the $4 \mathrm{~d}^{1} \mathrm{~S}_{0}-4 \mathrm{p}{ }^{1} \mathrm{P}_{1}$ line at $189 \AA$ 践 the $4 \mathrm{f}^{1} \mathrm{P}_{1}-4 \mathrm{~d}^{1} \mathrm{P}_{1}$ line at $226 \AA$. For the $4 \mathrm{f}^{1} \mathrm{P}_{1}-4 \mathrm{~d}^{1} \mathrm{P}_{1}$ 


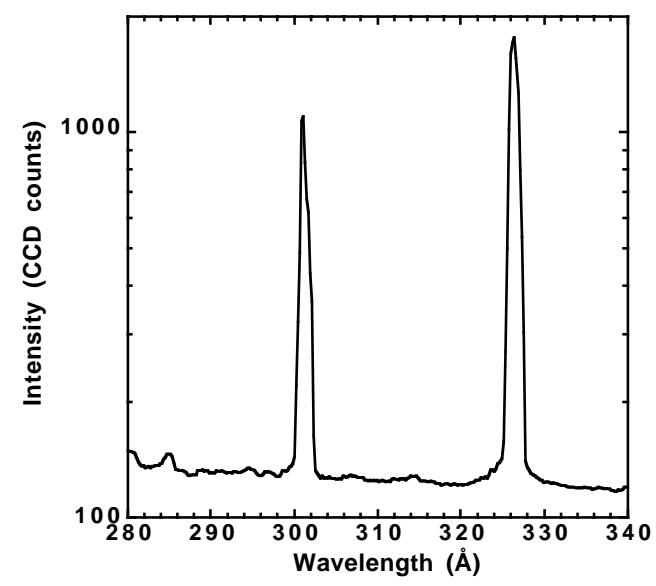

Fig. 1. Ne-like Ti spectrum showing lasing at 301 and $326 \AA ̊$.

line we discuss the role of the photopumping mechanism and present measured gain of $13 \mathrm{~cm}^{-1}$ for the $226 \AA$ Ni-like Mo line. We also present the calculated and measured wavelengths for the $4 \mathrm{f}^{1} \mathrm{P}_{1}-4 \mathrm{~d}{ }^{1} \mathrm{P}_{1}$ line for $\mathrm{Z}$ from 36 to 54 .

\section{PLASMA MODELING OF NE-LIKE TI EXPERIMENTS}

To model the Ti experiments done at the COMET facility we did LASNEX one dimensional (1D) computer simulations of a Ti slab illuminated by a $4.8 \mathrm{~J}, 800 \mathrm{psec}$ gaussian pulse followed $1.6 \mathrm{nsec}$ later by a $6 \mathrm{~J}, 1 \mathrm{psec}$ gaussian pulse from a 1.05 $\mu \mathrm{m} \mathrm{Nd}$ laser. The laser is focused to a $50 \mu \mathrm{m}$ wide by $1.2 \mathrm{~cm}$ long line. The $1.6 \mathrm{nsec}$ delay between the long and short pulse is peak to peak. This is similar to the conditions used to demonstrate lasing in Ne-like Ti and Ni-like Pd at the COMET laser

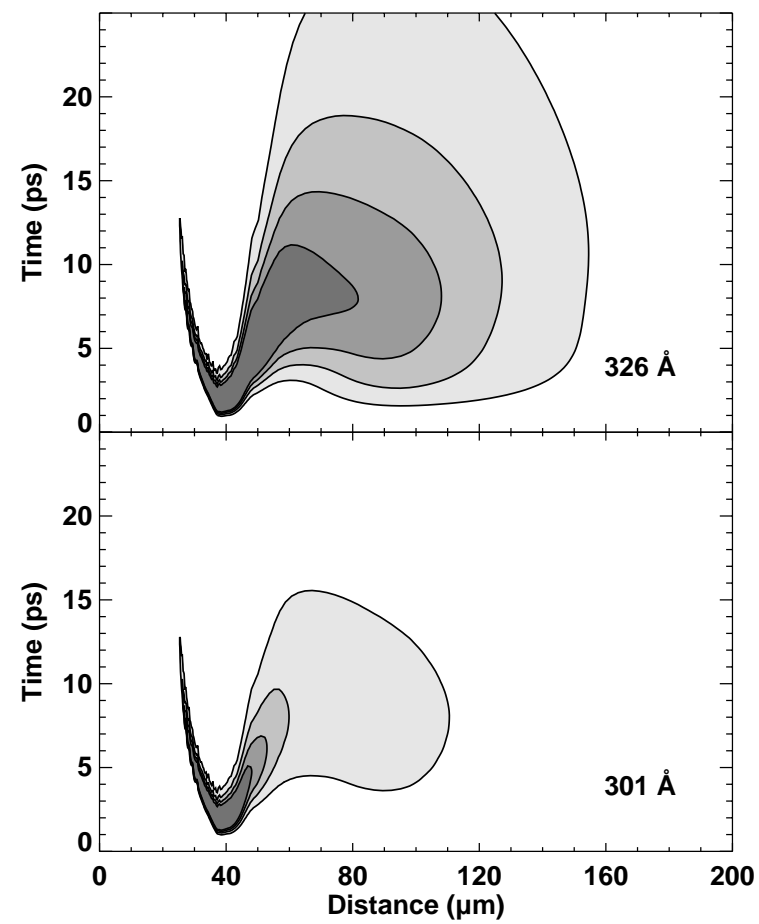

Fig. 2. Gain for the Ne-like Ti laser lines versus space and time. The short pulse laser drive peaks at 1 ps on the time axis. Contours represent gain $>80,60,40$, and $20 \mathrm{~cm}^{-1}$. 
facility. The LASNEX calculations include an expansion angle of 15 degrees in the dimension perpendicular to the primary expansion so as to simulate $2 \mathrm{D}$ effects.

The LASNEX calculated densities and temperatures are used as input to the XRASER code, which calculates the gains of the laser lines including radiation trapping effects for the four strong $3 \mathrm{~d}$ and $3 \mathrm{p} \_$- 2 resonance lines in Ne-like Ti. Bulk Doppler effects due to the expansion of the plasmas were also included. The Ti atomic model used by the XRASER code includes all 89 detailed levels for levels up to $\mathrm{n}=4$ in Ne-like Ti.

Four dominant laser lines are predicted and these can be divided into two classes. The $3 \mathrm{p}{ }^{1} \mathrm{~S}_{0} \ldots 3 \mathrm{~s}^{1} \mathrm{P}_{1}$ line at $326 \AA$ and the $3 \mathrm{p}^{1} \mathrm{~S}_{0} \_3 \mathrm{~s}^{3} \mathrm{P}_{1}$ line at $285 \AA$ share the same upper laser level which is populated by monopole collisional excitation of the Ne-like ground state. The $285 \AA$ line typically has half the gain of the $326 \AA$ line because its oscillator strength is smaller by a factor of 2 . The second set of lines are the $3 \mathrm{~d}^{1} \mathrm{P}_{1} \ldots 3 \mathrm{p}{ }^{1} \mathrm{P}_{1}$ line at $301 \AA$ and the $3 \mathrm{~d}^{1} \mathrm{P}_{1} \ldots 3 \mathrm{p}^{3} \mathrm{P}_{1}$ line at $314 \AA$. These lines share the same upper laser state which is populated primarily by photopumping on the strong $2 \mathrm{p}^{1} \mathrm{~S}_{0} \ldots \mathrm{d}^{1} \mathrm{P}_{1}$ transition. Both lines are predicted to lase, with the gain on the $314 \AA$ line about $25 \%$ lower because of its weaker oscillator strength. In experiments done at MBI and LLNL only the stronger monopole collisional and photopumped lines at 326 and $301 \AA$ lines are observed and we will only discuss those two lines further in this paper. Figure 1 shows a laser spectrum taken during an experiment at the COMET laser. One observes strong lasing on both the 326 and $301 \AA$ laser lines. While the $326 \AA$ line is very robust to changes in laser conditions the intensity of the $301 \AA$ line is quite sensitive to small changes and its intensity varies a lot from shot to shot.

Figure 2 shows contours of the gain versus space and time for the $326 \AA$ and $301 \AA$ laser lines for the nominal drive conditions described above. The short pulse laser peaks at $1 \mathrm{psec}$ on this time scale so the evolution during the long pulse is not shown. The horizontal axis gives the distance from the target surface in the direction of the plasma expansion. The gain contours represent gains greater than $80,60,40$, and $20 \mathrm{~cm}^{-1}$. The highest gain for the $326 \AA$ line exceeds $200 \mathrm{~cm}^{-1}$ at $40 \mu \mathrm{m}$, which is near the critical density surface, just after the time of peak illumination, 2 psec. This gain region is very small and short lived and has steep gradients which would make it difficult to propagate any significant distance. If one looks at the 2nd

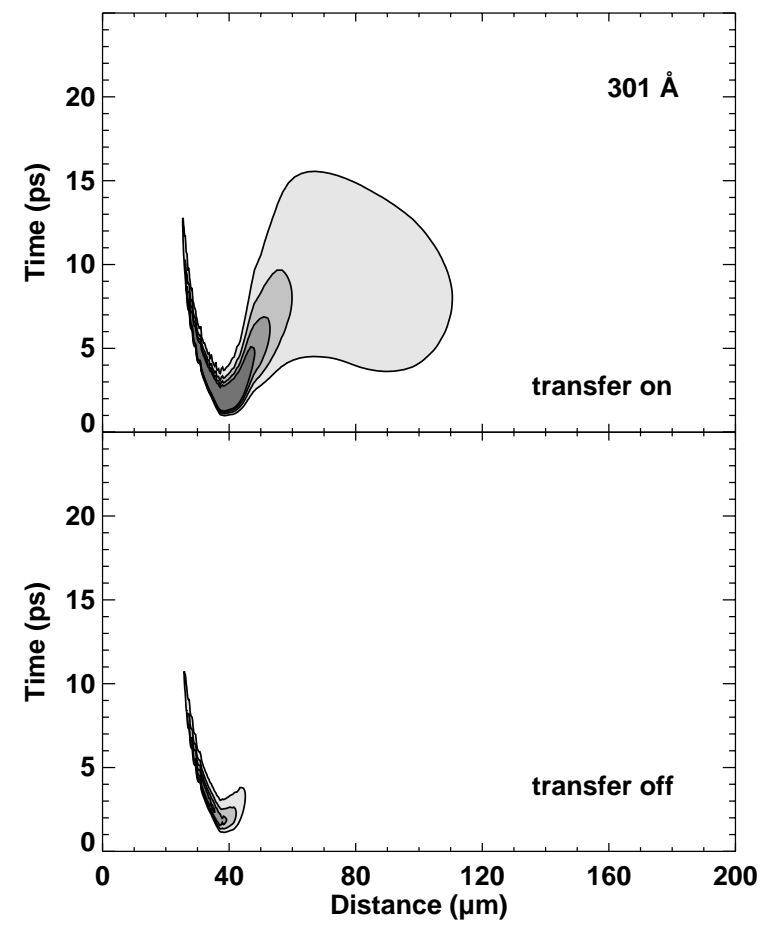

Fig. 3. Gain of the Ne-like Ti $301 \AA$ laser line with line transfer turned on and off versus space and time. The contours represent gain $>80,60,40$, and $20 \mathrm{~cm}^{-1}$. 
gain contour with $\mathrm{g}>60 \mathrm{~cm}^{-1}$ one can see that this region of large gain peaks about $80 \mu \mathrm{m}$ from the target surface at about 8 psec, which is long after the short pulse laser has turned off. The gain lasts about 10 psec and extends over $50 \mu \mathrm{m}$. For the $301 \AA$ laser line the gain region is somewhat shorter lived and has a smaller spatial extent which helps explain why this line is more sensitive to small changes in the experimental conditions. For both lines there is also a very narrow gain region which follows the critical density surface but that region is unlikely to allow laser propagation over any significant distance.

Normally, we would not expect gain on the $301 \AA$ line because its upper laser state is the $3 \mathrm{~d}^{1} \mathrm{P}_{1}$ level, which is depopulated by the rapid radiative decay to the ground state via the strongest resonance line. In this plasma, however, the $2 p$ ${ }^{1} \mathrm{~S}_{0} \ldots 3 \mathrm{~d}{ }^{1} \mathrm{P}_{1}$ line is optically thick and radiation trapping allows a very large radiation field to build up on this line and populate the $3 \mathrm{~d}^{1} \mathrm{P}_{1}$ upper laser state by the photopumping process (radiative excitation). To understand this mechanism, we did the XRASER calculations with the line transfer package turned off so that all the $n=2{ }_{-} n=3$ resonance lines are optically thin and the self photopumping process is absent. For the $326 \AA$ laser line, looking $80 \mu \mathrm{m}$ from the surface at 9 ps, there is a modest reduction of one-third in the gain. For the $301 \AA$ laser line, Fig. 3 shows contours of the gain with line transfer on and off. In contrast to the $326 \AA$ laser line, the gain of the $301 \AA$ laser line disappears without the photopumping process except for a very small region near the critical density surface.

We can better understand the role of the photopumping process by comparing the collisional and radiative rates from the Ne-like ground state to the $3 \mathrm{~d}{ }^{1} \mathrm{P}_{1}$ upper laser state versus distance from the target surface at $8.9 \mathrm{psec}$, as shown in Fig. 4. At $80 \mu \mathrm{m}$ from the surface the radiative rate is more than an order of magnitude larger than the collisonal excitation rate. The collisional rate falls very rapidly with the electron density while the radiative rate depends on the photon density. While collisional excitations create the photons, the large optical depth of the $2 \mathrm{p}^{1} \mathrm{~S}_{0}{ }_{-} 3 \mathrm{~d}^{1} \mathrm{P}_{1}$ line, ${ }_{-}=300$, allows photons which are created in the hot dense plasma near the surface and throughout the plasma to be trapped and contribute to the photopumping process.

One of the important parameters which affects the X-ray laser performance is the delay between the long and short pulse. Figure 5 shows the gain contours versus space and time for three different values of the delay, 0.6, 1.6, and 2.6 nsec. For the shortest delay of $0.6 \mathrm{nsec}$ the gain has a small temporal and spatial extent near the critical density surface just after the time of peak laser illumination. Given the large gradients in the plasma one would not expect to see any significant lasing in this case. For the longest delay of $2.6 \mathrm{nsec}$, the gain duration is much longer but has a narrow spatial extent. In contrast, for the 1.6-nsec delay, the gain has both a large spatial and temporal extent. The electron density gradients are also smallest for the 1.6-nsec case, which allows the $\mathrm{X}$-rays to propagate through the gain region and be amplified better. During the short pulse laser illumination the hydrodynamics does not change much and the main change in electron density is just due to ionization. For all three delays, the electron temperature becomes quite high, with peak values over $800 \mathrm{eV}$, so this is not a

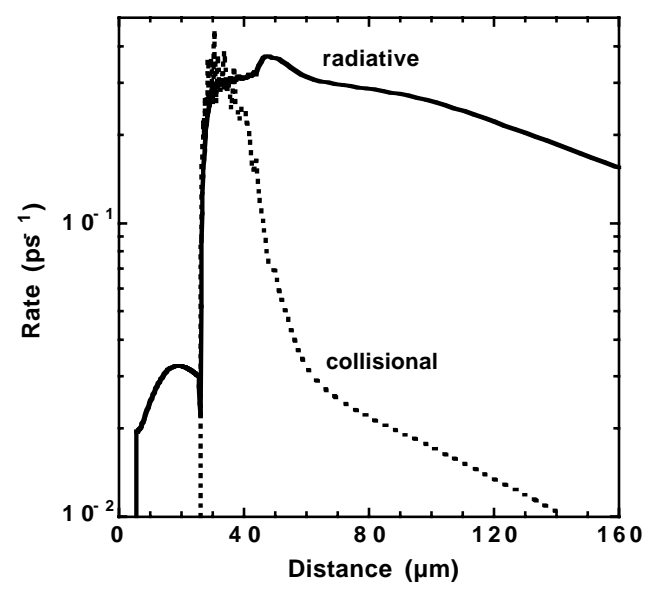

Fig. 4. Radiative and collisional rates for the $2 p{ }^{1} S_{0} \rightarrow 3 d{ }^{1} P_{1}$ transition versus distance from the target surface at $8.9 \mathrm{ps}$ show the photopumping process driving the gain for the $301 \AA$ laser line. 


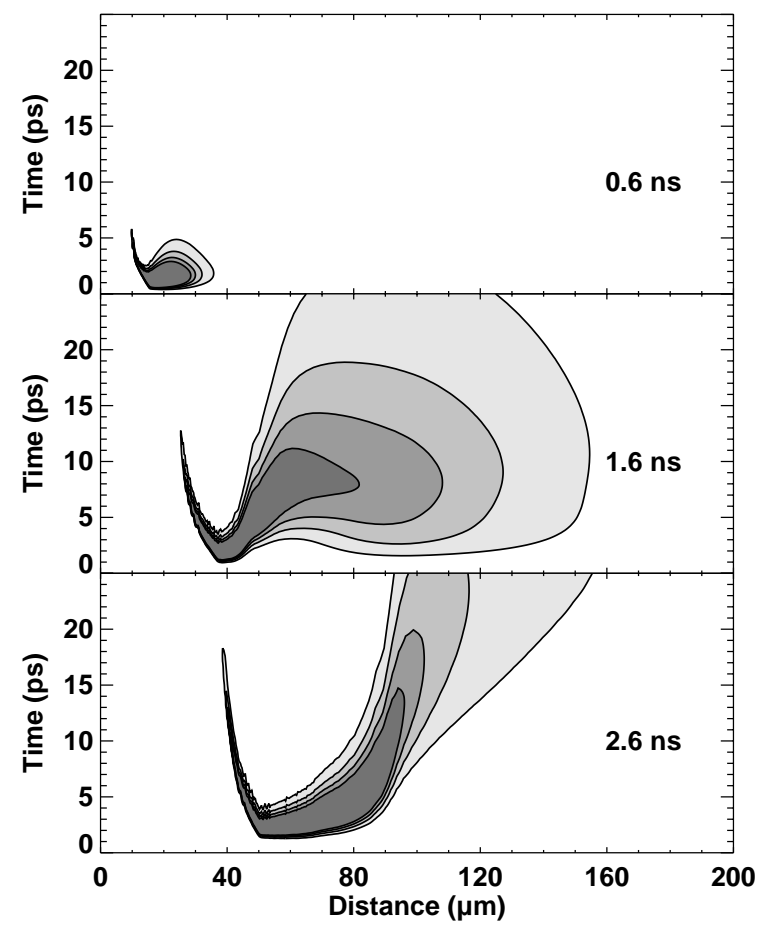

Fig. 5. Gain of the Ne-like Ti $326 \AA$ laser line versus space and time for three values of the delay between the long and short pulses. The short pulse laser peaks at $1 \mathrm{ps}$ on the time axis. Contours represent gain $>80,60,40$, and $20 \mathrm{~cm}^{-1}$.

limiting factor.

A big difference between the three cases is the ionization balance. Figure 6 shows the fraction of Ne-like Ti versus

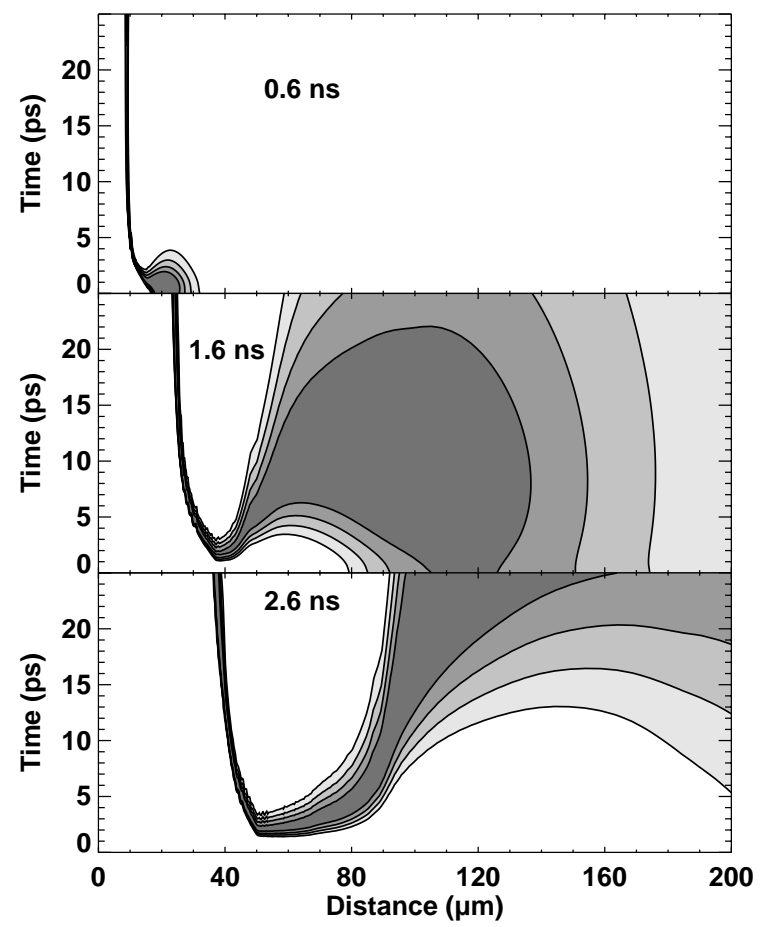

Fig. 6. Ne-like Ti ion fraction versus space and time for three values of the delay between the long and short pulses. Contours represent Ne-like fractions $>50,40,30$, and $20 \%$. 
space and time for the three delays. Recall that the short pulse laser peaks at $1 \mathrm{psec}$ on this time scale. The darkest contours represent Ne-like fraction greater than 50\% with the other contours being 40, 30, and 20\% fraction. For the 0.6-nsec delay the plasma tends to be overionized except at high densities. The long pulse over ionizes the plasma and the delay is too short to allow recombination to Ne-like. For the 2.6-nsec delay the plasma is underionized so that at time zero, before the short pulse laser is turned on, there is no Ne-like population except in the low-density region far from the gain region. In contrast, for the $1.6 \mathrm{nsec}$ delay there is a large Ne-like population before the short pulse laser heats the plasma. It appears that the best delay is one that prepares the plasma with a large Ne-like population. The short pulse laser then can quickly heat the electrons, which drives the collisional excitation rate, without allowing time for the plasma to overionize.

For the optimum 1.6-nsec delay we also did calculations with different short pulse energy. If we double the short pulse energy to $12 \mathrm{~J}$ the gain increases and the region of high gain expands in time and space. Halving the energy to $3 \mathrm{~J}$ reduces the gain and shrinks the gain region. This is consistent with the general observation that hitting a target harder makes it lase better. Given the long time duration for the gain compared with the 1 ps drive laser we did a calculation where we kept the energy of the short pulse laser at $6 \mathrm{~J}$ but increased the pulse duration to $5 \mathrm{ps}$. In that case we observed similar gain to the $1 \mathrm{ps}$ drive case but the time duration of the gain increases slightly and the spatial extent decreases slightly. This suggests that a longer drive pulse should work just fine and that energy on target is the more important issue since we are operating in a regime where the gain duration is longer than the pulse duration of the drive laser. The longer pulse would have the advantage of relaxing the requirements on implementing the traveling wave geometry in the experiments. For example the use of stepped mirrors or tilted compressor gratings to make the traveling wave geometry introduce non-ideal features that might be less of a problem with the use of the longer pulse drive.

To understand the effect of refraction on the X-ray laser output we return to the case of the optimum $1.6 \mathrm{nsec}$ delay. To study the refraction issue we consider a $1 \mathrm{~cm}$ long plasma which we illuminate from the end by a source which is weighted by the upper level population of the laser transition. We then do a ray tracing calculation with amplification to determine the near field and far field laser intensity versus space and time. For simplicity we assume traveling wave illumination by the short pulse laser. The laser output peaks at 8 psec with a FWHM duration of 1 psec. This shows that the gain region, which is contributing most to the output, is near $8 \mathrm{psec}$. If we now integrate over time and look at the laser intensity versus source position we observe a peak about $90 \mu \mathrm{m}$ from the target surface. These X-rays start at $90 \mu \mathrm{m}$ from the surface but are pointed inwards about $10 \mathrm{mrad}$ so that they sample gain even closer to the surface before they are refracted back out. If we look at the plasma $90 \mu \mathrm{m}$ from the surface at 8 psec the electron density is $8.2 \times 10^{19} \mathrm{~cm}^{-3}$ with an electron density gradient of $-6.5 \times 10^{21}$ $\mathrm{cm}^{-4}$. A $326 \AA$ A photon propagating through down a $1 \mathrm{~cm}$ length with this gradient would be bent by $15 \mu \mathrm{m}$. The refraction effect is quite small in this region, which no doubt accounts for why it contributes so much to the laser output.

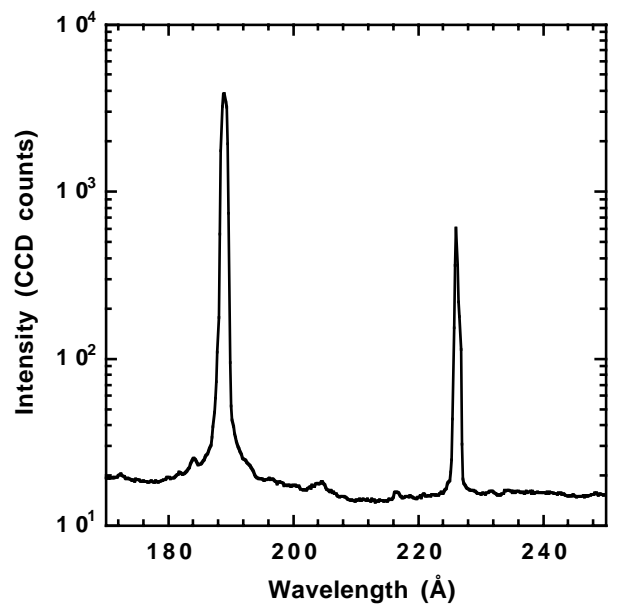

Fig. 7. Ni-like Mo spectrum showing lasing at 189 and $226 \AA ̊$. 


\section{NI-LIKE MO EXPERIMENTS}

We have also had great success making Ni-like ions lase under similar conditions as those used for the Ne-like $\mathrm{Ti}$ experiments at the COMET laser facility. In this section we will discuss our recent experiments on Ni-like Mo and in particular discuss the successful lasing observed on the $4 \mathrm{f}^{1} \mathrm{P}_{1}-4{ }^{1} \mathrm{P}_{1}$ photopumped line. To make Mo lase, we used a 600 FWHM pulse to preform and ionize the plasma followed by a 1 ps pulse to heat the plasma. The delay between the two pulses was $700 \mathrm{ps}$. A maximum energy of 5 joules was used in the short pulse and only 1 joule in the long pulse. The beams were focused to a $70 \mu \mathrm{m} \times 1.25 \mathrm{~cm}$ line on to the slab targets that vary in length up to $1 \mathrm{~cm}$. The long pulse was defocused by a factor of 2 to ensure better overlap between the beams from the long and short pulse. A five-segmented stepped mirror was used to provide travelling wave illumination of the target at the speed of light.

Figure 7 shows an on-axis Mo spectrum with the strong $4 \mathrm{~d}^{1} \mathrm{~S}_{0} \rightarrow 4 \mathrm{p}{ }^{1} \mathrm{P}_{1}$ line lasing at $189 \AA$ and the weaker $4 \mathrm{f}^{1} \mathrm{P}_{1} \rightarrow 4 \mathrm{~d}$ ${ }^{1} \mathrm{P}_{1}$ line lasing at $226 \AA$. The ratio between the lines is slightly distorted because the $189 \AA$ line is saturated on the detector. However the $226 \AA$ line lases very well and both lines completely dominate the background emission. Lasing on the $189 \AA$ $4 \mathrm{~d}^{1} \mathrm{~S}_{0} \rightarrow 4 \mathrm{p}{ }^{1} \mathrm{P}_{1}$ line in Ni-like Mo has been discussed in two recent papers [6,9] so we will not discuss this further here.

To measure the gain of the $226 \AA$ line we did a length study by using polished Mo targets that varied in length from 0.2 to $0.9 \mathrm{~cm}$. Figure 8 shows the intensity versus target length for this line from which we estimate a gain of $13 \mathrm{~cm}^{-1}$. There is no sign of saturation in this figure. In our modeling we estimate the saturation intensity of the $226 \AA$ line at $9 \mathrm{GW} \mathrm{cm}^{-2}$ under the plasma conditions at the time of peak gain. This is almost three times the saturation intensity estimated for the $189 \AA$ line and indicates that while the $226 \AA$ line is weaker and has lower gain it does have the potential to be the stronger line.

We have also observed lasing on the $4 \mathrm{f}^{1} \mathrm{P}_{1} \rightarrow 4 \mathrm{~d}^{1} \mathrm{P}_{1}$ line in Ni-like $\mathrm{Zr}$ and $\mathrm{Nb}$. The wavelengths of the measured laser lines with their uncertainties are given in Table 1 . While this line has not been observed directly in nonlasing plasmas, its wavelength can be calculated by using the measured values of the $3 d^{1} S_{0}-4 f{ }^{1} P_{1}$ resonance line together with the experimentally determined values for the energy of the $4 \mathrm{~d}^{1} \mathrm{P}_{1}$ lower laser state. This approach is described in Ref. 10 and the experimentally based wavelengths for $Z=37$ to 42 are given in Table 1 along with their uncertainties. For $Z=40$ to 42 these values agree with the values measured in the $X$-ray laser experiments within the experimental uncertainties. The maximum disagreement is $0.7 \AA$.

We calculated the wavelengths of the laser lines using the multi-configuration Dirac-Fock (MCDF) atomic physics code of Grant et al. in the extended average level (EAL) mode doing separate calculations for the $\mathrm{J}=1$ even and odd parity states. The energies of the $4 \mathrm{f}^{1} \mathrm{P}_{1}$ and $4 \mathrm{~d}^{1} \mathrm{P}_{1}$ level were subtracted to calculate the energy of the laser transition. We fit the difference between the energies calculated by the MCDF code and the experimentally determined energies for $Z=37$ to 42 to a straight line and then subtracted this curve from the MCDF calculation. For $\mathrm{Z}=42$ the correction was $0.77 \mathrm{eV}$. The wavelengths

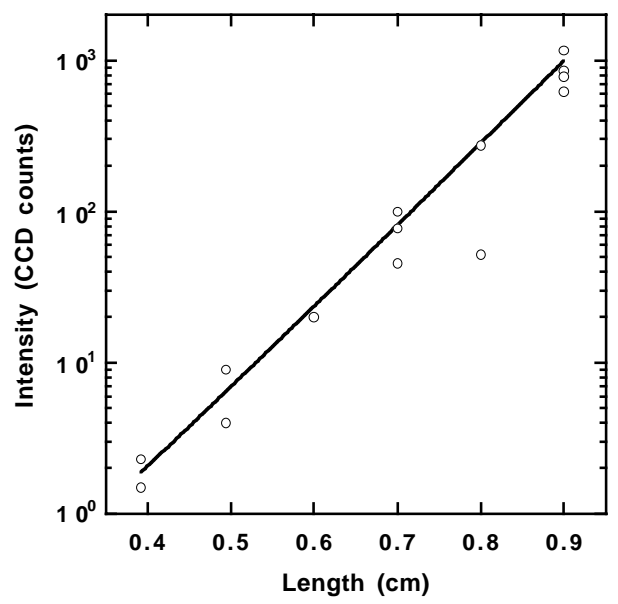

Fig. 8. Intensity versus length for the $226 \AA$ Ni-like Mo photopumped laser line. Measured gain is $13 \mathbf{c m}^{-1}$. 
Table 1. Wavelengths in $\AA$ of the $4 \mathrm{f}^{1} \mathrm{P}_{1} \rightarrow 4 \mathrm{~d}^{1} \mathrm{P}_{1}$ transition in Ni-like ions. Uncertainty of the last digit is given in parentheses.

\begin{tabular}{|c|c|c|c|}
\hline $\mathrm{Z}$ & $\begin{array}{c}\text { Laser } \\
\text { measurement }\end{array}$ & $\begin{array}{c}\text { Calculated from } \\
\text { experimental energies }\end{array}$ & $\begin{array}{c}\text { Predicted } \\
\text { wavelengths }\end{array}$ \\
\hline 36 & & & 443.4 \\
\hline 37 & & $383.9(2)$ & 383.6 \\
\hline 38 & & $337.1(2)$ & 337.3 \\
\hline 39 & & $300.4(2)$ & 300.6 \\
\hline 40 & $271.0(2)$ & $270.8(3)$ & 270.9 \\
\hline 41 & $246.4(3)$ & $247.0(5)$ & 246.5 \\
\hline 42 & $226.0(3)$ & $226.7(5)$ & 226.1 \\
\hline 43 & & & 208.9 \\
\hline 44 & & & 194.1 \\
\hline 45 & & & 181.3 \\
\hline 46 & & & 170.1 \\
\hline 47 & & & 160.4 \\
\hline 48 & & & 151.8 \\
\hline 49 & & & 144.7 \\
\hline 50 & & & 135.5 \\
\hline 51 & & & 129.6 \\
\hline 52 & & & 124.0 \\
\hline 53 & & & 118.8 \\
\hline 54 & & & 114.0 \\
\hline
\end{tabular}

predicted by this method are given in Table 1 for $\mathrm{Z}=36$ to 54 . We believe these values are accurate to better than $1 \AA$. Similar calculations done for the $4 \mathrm{~d}^{1} \mathrm{~S}_{0} \rightarrow 4 \mathrm{p}{ }^{1} \mathrm{P}_{1}$ line are described in Ref. 9.

To understand the photopumping process we modeled the Mo plasma using the LASNEX and XRASER codes as described above for Ti. In the calculations, the gain of the $226 \AA$ laser line peaks about 1 ps after the peak of the short pulse at $27 \mu \mathrm{m}$ from the target surface with a peak gain of $160 \mathrm{~cm}^{-1}$. The very high gain falls by half within $2 \mathrm{ps}$ but gain greater than $20 \mathrm{~cm}^{-1}$ persists for $8 \mathrm{ps}$ after the short pulse. In Fig. 9 we show rates typical for the plasma at $5 \mathrm{ps}$ after the peak of the short pulse at $50 \mu \mathrm{m}$ from the target surface. The radiative excitation rate due to photopumping is the dominant rate and is an order of magnitude larger than the collisional excitation rate which is populating the $4 \mathrm{f}$ upper laser level. The optical depth of the $3 \mathrm{~d}^{1} \mathrm{~S}_{0}$ - $4 \mathrm{f}{ }^{1} \mathrm{P}_{1}$ resonance line is 100 and the line strength is 0.0163 photons per mode. The gain of the $226 \AA$ Mo line is $37 \mathrm{~cm}^{-1}$ with an electron temperature of $295 \mathrm{eV}$, an ion temperature of $47 \mathrm{eV}$, ion density of $4.42 \times 10^{18} \mathrm{~cm}^{-3}$ and an electron density of $6.30 \times 10^{19} \mathrm{~cm}^{-3}$. The gain profile has a spatial FWHM width of $60 \mu \mathrm{m}$. The gradient in the electron density is $-1.69 \times 10^{22} \mathrm{~cm}^{-4}$. For a $226 \AA \mathrm{X}$-ray, this gradient would refract the X-ray by only $19 \mu \mathrm{m}$ over a 1 -cm propagation length. The collision rates shown for the lower laser state are net rates out of the level. In the absence of photopumping the gain disappears and goes slightly negative. 


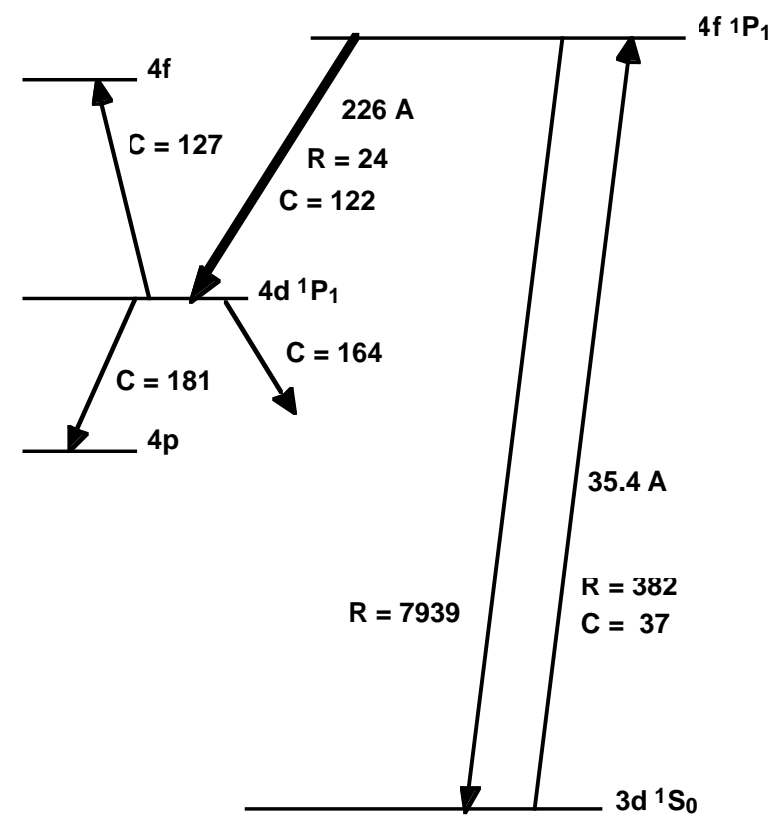

Fig. 9. Energy level diagram showing the collisional ( $C$ ) and radiative ( $R$ ) rates ( nsec $^{-1}$ ) which drive the $226 \AA$ Ni-like Mo $4 \mathrm{f}^{1} \mathrm{P}_{1} \rightarrow 4 \mathrm{~d}^{1} \mathrm{P}_{1} \mathrm{X}$-ray laser line. Lasing is driven by the strong photopumping from the $3 d{ }^{1} S_{0}$ ground state to the $4 f^{1} P_{1}$ upper laser level.

\section{CONCLUSIONS}

In this work we model recent experiments done using the COMET laser at LLNL to illuminate $1 \mathrm{~cm}$ long slab targets of Ti with a $4.8 \mathrm{~J}, 800$ ps prepulse followed 1.6 nsec later by a $6 \mathrm{~J}, 1$ psec drive pulse. The LASNEX code is used to calculate the hydrodynamic evolution of the plasma and provide the temperatures and densities to the XRASER code, which then does the kinetics calculations to determine the gain. The temporal and spatial evolution of the plasma is studied both with and without radiation transport included for the $3 \mathrm{~d}$ and $3 \mathrm{~s}_{-} 2 \mathrm{p}$ Ne-like Ti resonance lines. Large regions with gain greater than $80 \mathrm{~cm}^{-1}$ are predicted for the $3 \mathrm{p}^{1} \mathrm{~S}_{0} \ldots 3 \mathrm{~s}^{1} \mathrm{P}_{1} \mathrm{Ne}$-like Ti laser line at $326 \AA$. Given the large gain and large gradients in these plasmas, we do propagation calculations including refraction to understand which regions have the right combination of high gain and low gradients to contribute to the X-ray laser output. Calculations are also presented using different delays between the long and short pulse and different widths for the short pulse to provide better insight for optimizing the laser output. The delay appears to play a large role in preparing the plasma in the correct ionization stage prior to the short pulse heating the plasma. In addition to the standard $326 \AA$ laser line, high gain is also predicted and observed for the $3 \mathrm{~d}^{1} \mathrm{P}_{1}{ }_{1} 3 \mathrm{p} \quad{ }^{1} \mathrm{P}_{1}$ laser line at $301 \AA$ in Ne-like Ti. We present calculations with and without radiation transport included on the strong $3 \mathrm{~d}^{1} \mathrm{P}_{1}{ }_{2} 2 \mathrm{p} \quad{ }^{1} \mathrm{~S}_{0}$ resonance line to better understand this self-photopumping effect.

For Ni-like Mo we demonstrate lasing on both the $4 \mathrm{~d}^{1} \mathrm{~S}_{0}-4 \mathrm{p}{ }^{1} \mathrm{P}_{1}$ line at $189 \AA$ and the $4 \mathrm{f}^{1} \mathrm{P}_{1}-{ }^{4 \mathrm{~d}}{ }^{1} \mathrm{P}_{1}$ line at $226 \AA$. For the $4 \mathrm{f}^{1} \mathrm{P}_{1}-4 \mathrm{~d}^{1} \mathrm{P}_{1}$ line we explain how the photopumping mechanism drives the gain of this line and present measured gain of $13 \mathrm{~cm}^{-1}$ for this line. We also present the calculated and measured wavelengths for the $4 \mathrm{f}^{1} \mathrm{P}_{1}-4 \mathrm{~d}^{1} \mathrm{P}_{1}$ line for $\mathrm{Z}$ from 36 to 54 . 


\section{ACKNOWLEDGEMENTS}

The authors would like to thank Richard Ward, Mark Eckart, Andi Hazi, and Luiz Da Silva for their support. Yuelin Li acknowledges support from Hector Baldis of the Institute for Laser Science and Applications (ILSA). The authors would like

to thank A. N. Ryabtsev and S. S. Churilov for their valuable advice and Jim Hunter for technical support for the experiments. Work performed under the auspices of the U. S. Department of Energy by the Lawrence Livermore National Laboratory under contract No.W-7405-ENG-48.

\section{REFERENCES}

[1] J. Nilsen, B. J. MacGowan, L. B. Da Silva, and J. C. Moreno, Phys. Rev. A 48, 4682 (1993).

[2] J. C. Moreno, J. Nilsen, and L. B. Da Silva, Opt. Comm. 110, 585 (1994).

[3] J. Nilsen and J. C. Moreno, Phys. Rev. Lett. 74, 3376 (1995).

[4] P. V. Nickles, V. N. Shlyaptsev, M. Kalachnikov, M. Schnuerer, I. Will, and W. Sandner, Phys. Rev. Lett. 78, 2748 (1997).

[5] J. Dunn, A. L. Osterheld, R. Shepherd, W. E. White, V. N. Shlyaptsev, and R. E. Stewart, Phys. Rev. Lett. 80, 2825 (1998).

[6] J. Dunn, J. Nilsen, A. L. Osterheld, Y. L. Li, and V. N. Shlyaptsev, Opt. Lett. 24, 101 (1999).

[7] J. Nilsen, Phys. Rev. A 53, 4539 (1996).

[8] J. Nilsen, Phys. Rev. A 55, 3271 (1997).

[9] Y. L. Li, J. Nilsen, J. Dunn, A. L. Osterheld, A. Ryabtsev, and S. Churilov, Phys. Rev. A 58, R2668 (1998).

[10] A. N. Ryabtsev, S. S. Churilov, J. Nilsen, Y. L. Li, J. Dunn, and A. L. Osterheld, "Additions to the analysis of the Nilike ion spectra," Opt. Spectrosc. (in press 1999). 